\title{
Power Threshold Due to Parametric Gain in Dispersion-Mapped Communication Systems
}

\author{
Paolo Serena and Alberto Bononi
}

\begin{abstract}
Parametric gain (PG) is investigated in conventional terrestrial transmission systems at $10 \mathrm{~Gb} / \mathrm{s}$. The authors present new results that show how at large signal transmitted power the $P G$ effect strongly differs from the predictions of the standard smallsignal approach, which neglects the noise quadratic and higher order terms in the nonlinear Schrödinger equation. The authors give a new explicit expression of the maximum transmittable power in a dispersion-mapped transmission system impaired by PG and show that such power threshold is due to the inflation of the low frequency portion of the in-phase noise spectrum.
\end{abstract}

Index Terms-Karhunen-Loéve expansion, Kerr effect, modulation instability, parametric gain (PG).

\section{INTRODUCTION}

$\mathbf{P}$ ARAMETRIC gain (PG) is a nonlinear effect due to the interaction between a strong pump signal and amplified spontaneous emission (ASE) noise that produces a colored ASE at the receiver, with a correlation between its real (in-phase) and imaginary (quadrature) components [1], [2].

The standard analytical tool for the study of PG is a linearization approach to the nonlinear Schrödinger equation (NLSE) starting from a continuous-wave $(\mathrm{CW})$ pump, set at the power level of marks in an ON-OFF keying (OOK) transmission, plus a small ASE perturbation field [1], [2]. The linearization is obtained by dropping all quadratic and higher order terms of the perturbating ASE, thus obtaining a linear propagation equation of the real and imaginary ASE components. Such a vector differential equation has a solution in the form of an end-to-end system matrix relating the input and output ASE fields [1], [2]. Being the output ASE is a linear filtering of the input, ASE's statistics remain Gaussian at the photoreceiver.

Using the PG linearized model and the Gaussian approximation for bit-error-rate (BER) evaluation, it has been shown in [3] that for dispersion-mapped systems operating in the average normal dispersion regime PG can actually reduce the deleterious impact of ASE noise when compared with linear propagation, while in the anomalous dispersion regime PG always degrades the system performance.

It has also been shown that whenever the in-phase and quadrature ASE components are strongly correlated, the Gaussian approximation for BER evaluation fails and well-known techniques for the performance evaluation of quadratic detectors in Gaussian noise, based on a Karhunen-Loéve (K-L) expansion of the ASE have been adapted to the PG case [4].

Manuscript received July 1, 2002; revised August 5, 2002.

The authors are with the Dipartimento di Ingegneria dell'Informazione, Università degli studi di Parma, 43100 Parma, Italy (e-mail: serena@tlc.unipr.it; bononi@ tlc.unipr.it).

Digital Object Identifier 10.1109/LPT.2002.803893.
In this letter, we show that the PG linearized approach is valid only at small pump power levels. At large pump powers, we prove that the PG linearized solution differs from the exact one mostly because the received ASE in-phase power spectral density (PSD) inflates in the frequency region around $\omega=0$ well above the value predicted by the linearized model, as already noted in [5]. Such in-band "inflation" of the in-phase PSD, which is present both in the normal and in the anomalous dispersion regime, is the major cause of system degradation at large transmitted signal power, since it contributes to the dominant signal-ASE beat noise and is due to the quadratic and higher order ASE terms which are dropped in the linearized model. Note that, in the context of modulational instability pulsed lasers, the ASE contribution around $\omega=0$ appears as a background field between pulses [6].

In order to accurately evaluate the BER even in the largepump regime, we have: 1) estimated the received ASE PSD by propagating a $\mathrm{CW}$ plus long sequences of ASE samples using the split step Fourier method (SSFM), and then averaged with the averaged periodogram method [7] and 2) by using such accurate ASE PSD, and by pretending that the received ASE remains Gaussian distributed, we have applied the $\mathrm{K}-\mathrm{L}$ method for the BER evaluation.

As a result, we have observed a BER power threshold beyond which transmission is disrupted. For several dispersion maps such a threshold can be significantly lower than the threshold due to self-phase modulation acting on the OOK signal alone. Such a threshold cannot be predicted by the PG linearized model.

Another important result of this paper is the stochastic analysis of the nonlinear PG model, where we keep the quadratic ASE terms in the NLSE, which leads to an analytic formula of the in-phase ASE PSD at $\omega=0$. We observed that the BER power threshold is always quite close to the pump power at which the in-phase ASE PSD at $\omega=0$ doubles its value with respect to the linear regime. We call such power value the PG power threshold. The main implications of the explicit PG power threshold formula are also discussed.

\section{PG LINEARIZED MODEL AND EXTENSION}

When the fiber input is composed of a CW signal $(\sqrt{P})$ plus a small perturbation field $a(z, t)=a_{r}(z, t)+j a_{i}(z, t)$, with $|a|^{2} \ll P$, the NLSE is usually linearized in the frequency domain as [2]

$$
\left\{\begin{array}{l}
\frac{\partial \tilde{a}_{r}}{\partial z}=\frac{\beta_{2}}{2} \omega^{2} \tilde{a}_{i} \\
\frac{\partial \tilde{a}_{i}}{\partial z}=-\frac{\beta_{2}}{2} \omega^{2} \tilde{a}_{r}-2 \gamma P \tilde{a}_{r} e^{-\alpha z}
\end{array}\right.
$$




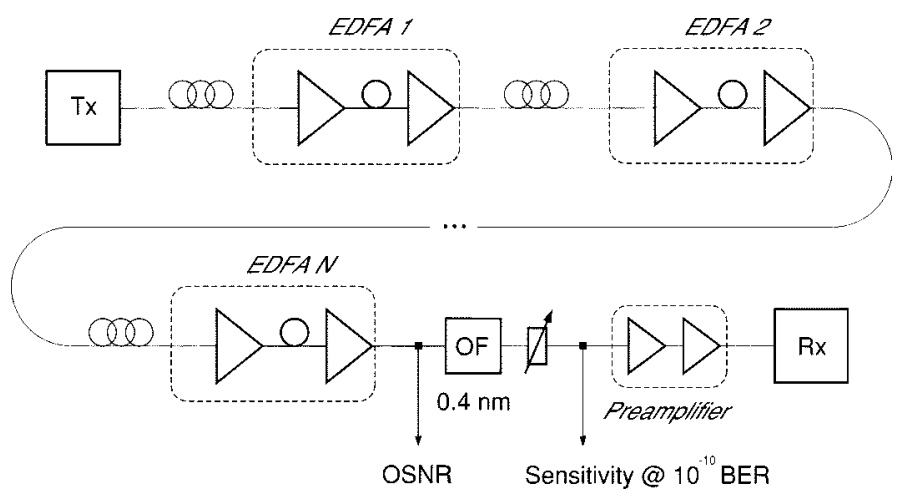

Fig. 1. Schematic diagram of the dispersion-mapped optical system with $N=$ 5 spans.

where $\tilde{a}_{r}$ and $\tilde{a}_{i}$ are the Fourier transforms of $a_{r}$ and $a_{i}$, respectively, $\alpha$ is the fiber attenuation, $\beta_{2}$ the fiber dispersion parameter, and $\gamma$ the nonlinear coefficient. From (1) we conclude that, at $\omega=0, \tilde{a}_{r}$ is invariant along $z$, and thus so is the received in-phase PSD. However, we will show next that at large CW powers the received ASE PSD at $\omega=0$ actually inflates well above its input value. The reason is that the quadratic and higher order terms which were dropped in the derivation of (1) cannot be neglected anymore. If also quadratic terms are included, (1) in the time domain becomes

$$
\left\{\begin{array}{l}
\frac{\partial a_{r}}{\partial z}=-\frac{\beta_{2}}{2} \frac{\partial^{2} a_{i}}{\partial t^{2}}+2 \gamma \sqrt{P} a_{r} a_{i} e^{-\alpha z} \\
\frac{\partial a_{i}}{\partial z}=\frac{\beta_{2}}{2} \frac{\partial^{2} a_{r}}{\partial t^{2}}-\gamma\left(2 P a_{r}+3 \sqrt{P} a_{r}^{2}+\sqrt{P} a_{i}^{2}\right) e^{-\alpha z}
\end{array}\right.
$$

This equation is our starting point for the derivation of the analytical formula of the PG power threshold.

\section{System IMPACT OF PG}

We have evaluated the impact of PG on a $10-\mathrm{Gb} / \mathrm{s} 5 \times 100-\mathrm{km}$ terrestrial transmission system, shown in Fig. 1, in terms of sensitivity penalty with respect to back-to-back transmission at BER $=10^{-10}$. The system is similar to the one experimentally investigated in [5], although it works at an optical signal to noise ratio OSNR $=21 \mathrm{~dB}$ over $0.1 \mathrm{~nm}$ in the absence of $P G$, and the transmission fiber is a nonzero dispersion shifted $\left(\mathrm{NZDSF}^{-}\right)$fiber, with dispersion $D=-2.6 \mathrm{ps} / \mathrm{nm} / \mathrm{km}$, attenuation $\alpha=0.2 \mathrm{~dB} / \mathrm{km}$, and nonlinear coefficient $\gamma=2.1$ $\mathrm{W}^{-1} \cdot \mathrm{km}^{-1}$. The in-line amplifiers are dual stage. Sandwiched between the stages, a standard single-mode fiber (SMF) of suitable length and with small enough input power provides the desired residual dispersion, with negligible nonlinearity.

In order to highlight the inaccuracy of the standard small-signal PG model at large transmitted powers, the correct ASE PSD, which includes the effect of quadratic and higher order terms in the NLSE, was estimated by the SSFM using the averaged periodogram method. Fig. 2 shows the in-phase and quadrature ASE PSD, normalized to their value without PG, both at a "small" $\mathrm{CW}$ power $P=11 \mathrm{dBm}$ (a), and at a "large" power $P=15 \mathrm{dBm}(\mathrm{b})$, evaluated at a residual dispersion

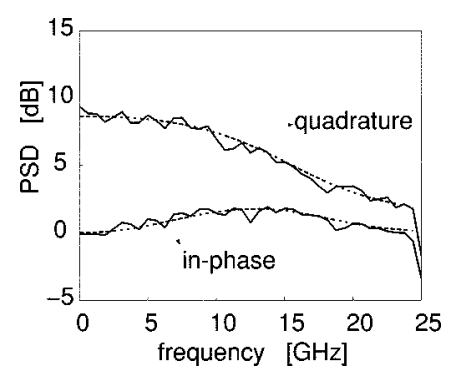

(a)

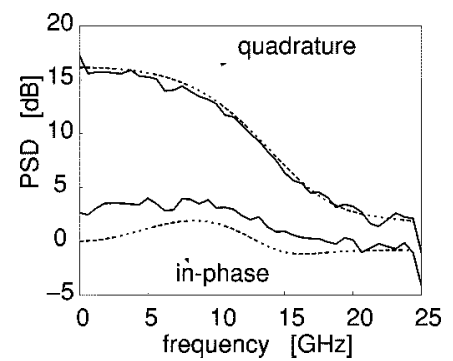

(b)

Fig. 2. Normalized in-phase and quadrature ASE PSD: (a) $P=11 \mathrm{dBm}$, (b) $P=15 \mathrm{dBm}$. Five-span NZDSF $-/ \mathrm{SMF}^{\mathrm{map}}$ at $D_{R}=0 \mathrm{ps} / \mathrm{nm}$. Averaged periodogram (solid line); PG small-signal solution (dashed line).

$D_{R}=0 \mathrm{ps} / \mathrm{nm}$. In the dashed line we also report the ASE PSD obtained by solving the PG linearized model (1) [2]. We note that, while at small powers the PSD evaluated with the PG linearized model coincides with the estimated one, at large CW powers the PG linearized model underestimates the in-phase PSD on a bandwidth of several gigahertz. We also note that the quadrature ASE PSD remains instead essentially unchanged on a log scale. Since the beat between signal and in-phase ASE mostly contributes to the noise at the decision gate [3], we expect that such low-frequency inflation strongly influences the system performance at large powers.

Since the standard Gaussian approximation gives inaccurate results in the presence of colored noise [4], we evaluated the system BER using a $\mathrm{K}-\mathrm{L}$ expansion of the received noise. In the numerical computation we used an extension of the method described in [8], which is simpler to implement than that in [4], since it avoids the explicit computation of the K-L eigenfunctions. In applying the $\mathrm{K}-\mathrm{L}$ method, we used the fact that ASE noise statistics after transmission are still approximately Gaussian and used its estimated PSD obtained by the SSFM. The in-phase PSD inflation changes the sensitivity penalty at large powers, as can be seen from Fig. 3. The top row shows the sensitivity penalty versus peak transmitted power, evaluated using the estimated ASE PSD (solid line) and the ASE PSD from the PG linearized model (dotted line), with overall residual dispersion $D_{R}=0 \mathrm{ps} / \mathrm{nm}$ (left plot) and $D_{R}=+500 \mathrm{ps} / \mathrm{nm}$ (right plot). The BER and thus the sensitivity penalty were evaluated by including the self-phase modulation (SPM) effect on the OOK signal. In the dashed lines we also report for comparison the penalty when considering SPM for the signal and neglecting PG, so that ASE remains spectrally flat, or white. From the figure, we observe that for both considered values of residual dispersion there are some decibels of shift in power threshold 

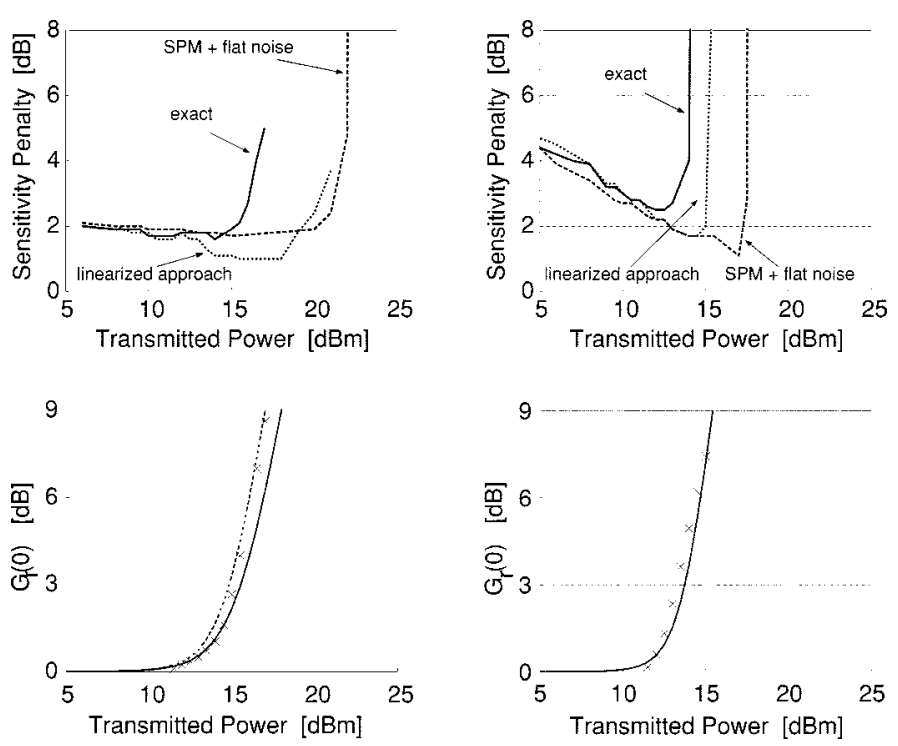

Fig. 3. (Top row) Sensitivity penalty versus peak transmitted power: exact PG+SPM (solid line), linearized PG+SPM (dotted line), white ASE+SPM (dashed line). (Bottom row) Normalized in-phase ASE PSD at $\omega=0$ versus peak transmitted power: analytical solution of (2) (solid line), (3) (dashed line), averaged periodogram method (crosses). Five-span NZDSF-/SMF map, with $D_{R}=0 \mathrm{ps} / \mathrm{nm}$ (left plots) and $D_{R}=+500 \mathrm{ps} / \mathrm{nm}$ (right plots).

when PG is considered, while the curves evaluated using either no PG or the PG linearized approach fail to predict the correct threshold value.

The bottom plots show the normalized in-phase ASE PSD at $\omega=0, G_{r}(0)$, versus peak transmitted power, evaluated by both stochastic analysis of the nonlinear system (2) (solid lines) and by direct simulation with the SSFM (crosses). We verified that stochastic analysis and simulation match well down to OSNR values of at least $10 \mathrm{~dB}$. We also clearly note the strong correlation between the power threshold in the sensitivity penalty in the top graphs and the one in $G_{r}(0)$ shown in the bottom graphs. The stochastic analysis is based on the first-order regular perturbation solution of (2) [9]. After involved computations of higher order joint moments of Gaussian processes, it yields the ASE $P S D$ in integral form, and only at $\omega=0$.

For the solid line curve in the case $D_{R}=0$ we have found an explicit approximation of the integral form for systems having a number of spans $N>2$, which is plotted in dashed line in Fig. 3

$$
G_{r}(0)=1+\frac{\sqrt{2} \gamma^{4} l_{\text {eff }}^{2} P^{4}}{\Delta \nu \operatorname{OSNR} \sqrt{\left|\beta_{2}\right|} \alpha^{3 / 2}} \cdot \Lambda(N)
$$

where $l_{\text {eff }}$ is the fiber effective length, $\Delta \nu$ the frequency band over which the OSNR is evaluated, and $\Lambda(N)$ is a function that accounts for the cumulative effect of the propagation in the $N$ span system

$$
\Lambda(N) \triangleq \frac{(N-1)(N-2)\left(20 N^{3}-48 N^{2}+31 N-21\right)}{960 N} .
$$

The $P^{4}$ term causes a very fast increase of the PSD as power approaches the PG threshold, which we nominally set to the value $P_{t h}$ at which $G_{r}(0)=2$ :

$$
P_{\text {th }} \triangleq\left(\frac{\sqrt{\left|\beta_{2}\right|} \alpha^{3 / 2} \Delta \nu \mathrm{OSNR}}{\sqrt{2} \gamma^{4} l_{\mathrm{eff}}^{2} \Lambda(N)}\right)^{1 / 4}
$$

We note that such a PG power threshold depends on the magnitude of the dispersion of the transmission fiber and not on its sign. It also depends on the OSNR level and the number of spans $N$. The presence of a nonzero residual dispersion can shift the power threshold by a few decibels, according to the map. When $D_{R} \neq 0$, we could not find a simple approximate explicit form of the integral form of $G_{r}(0)$.

\section{CONCLUSION}

We evaluated the sensitivity penalty for a $10-\mathrm{Gb} / \mathrm{s}$ dispersion mapped terrestrial system, with zero and positive residual dispersion. We proved that a transmitted power threshold exists, beyond which PG prevents error free transmission. We showed that such a PG threshold is connected with the inflation of the low-frequency part of the in-phase ASE PSD and cannot be predicted by the existing linearized models for PG. Although large threshold levels result from our numerical example, obtained at a large $O S N R$, from formula (5) we know such thresholds substantially decrease to "practical" levels at a lower OSNR and/or larger $N$, such as in longer systems or systems with larger interamplifier losses.

\section{ACKNOWLEDGMENT}

The authors would like to acknowledge the numerous stimulating discussions with S. Bigo and J. C. Antona of Alcatel CRC, Marcoussis, France.

\section{REFERENCES}

[1] M. Karlsson, "Modulation instability in lossy optical fibers," J. Opt. Soc. Amer. B, vol. 12, pp. 2071-2078, Nov. 1995.

[2] A. Carena, V. Curri, R. Gaudino, P. Poggiolini, and S. Benedetto, "New analytical results on fiber parametric gain and its effects on ASE noise," IEEE Photon. Technol. Lett., vol. 9, pp. 535-537, Apr. 1997.

[3] R. Hui, M. O'Sullivan, A. Robinson, and M. Taylor, "Modulation instability and its impact in multispan optical amplified IMDD systems: Theory and experiments," J. Lightwave Technol., vol. 15, pp. 1071-1082, July 1997.

[4] G. Bosco, A. Carena, V. Curri, R. Gaudino, P. Poggiolini, and S. Benedetto, "A novel analytical method for the BER evaluation in optical systems affected by parametric gain," IEEE Photon. Technol. Lett., vol. 12, pp. 152-154, Feb. 2000.

[5] G. Bellotti, S. Bigo, P. Serena, and A. Bononi, "Impact of parametric gain on $10 \mathrm{~Gb} / \mathrm{s}$ dispersion-managed transmissions," in Proc. ECOC'00, vol. P3.12, Sept. 2000, pp. 213-215.

[6] S. Coen and M. Haelterman, "Impedance-matched modulation instability laser for background-free pulse train generation in the Thz range," Opt. Commun., vol. 146, pp. 339-346, Jan. 1998.

[7] R. S. A. V. Oppenheim, Discrete-Time Signal Processing, 2nd ed. New York: Prentice Hall, 1999.

[8] E. Forestieri, "Evaluating the error probability in lightwave systems with chromatic dispersion, arbitrary pulse shape and pre- and postdetection filtering," J. Lightwave Technol., vol. 18, pp. 1493-1503, Nov. 2000.

[9] A. Vannucci, P. Serena, and A. Bononi, "The RP method: A new tool for the iterative solution of the nonlinear Schrödinger equation," J. Lightwave Technol., vol. 20, pp. 1102-1112, July 2002, to be published. 